\title{
Physica $C$
}

March 15th, 2014

\section{Epitaxial strain effect in perovskite $R E \mathrm{NiO}_{3}$ films $(R E=\mathrm{La}-\mathrm{Eu})$ prepared by metal organic decomposition}

\author{
Ai Ikeda ${ }^{\text {a,b }}$, Takaaki Manabe ${ }^{\mathrm{c}}, *$ Michio Naito ${ }^{\mathrm{a}}$ \\ ${ }^{\mathrm{a}}$ Department of Applied Physics, Tokyo University of Agriculture and Technology \\ Naka-cho 2-24-16, Koganei, Tokyo 184-8588, Japan \\ ${ }^{b}$ Research Fellow of the Japan Society for the Promotion of Science \\ ${ }^{\mathrm{c}}$ National Institute of Advanced Industrial Science and Technology (AIST) \\ Higashi 1-1-1, Tsukuba, Ibaraki 305-8565, Japan
}




\begin{abstract}
We report the synthesis of perovskite $R E \mathrm{NiO}_{3}$ films $(R E=\mathrm{La}, \mathrm{Pr}, \mathrm{Nd}, \mathrm{Sm}$, and $\mathrm{Eu})$ by metal organic decomposition (MOD). The $R E \mathrm{NiO}_{3}$ family is an ideal system for studying the metal-insulator transition due to the simplicity of the materials. One of the drawbacks is that the bulk synthesis of the $R E \mathrm{NiO}_{3}$ requires processing at high oxygen pressures to stabilize $\mathrm{Ni}^{3+}$. Fundamentally, MOD is similar to solid-state reaction, but it turned out that the MOD synthesis tends to stabilize $\mathrm{RENiO}_{3}$ without the need for high oxygen pressure. The films prepared by MOD show high crystallinity and low resistivity. Furthermore, we have investigated the epitaxial strain effect and observed a dramatic effect in $\mathrm{PrNiO}_{3}$ and $\mathrm{NdNiO}_{3}$ films on $\mathrm{LaAlO}_{3}$ substrates. The metal-insulator transition in the $\mathrm{PrNiO}_{3}$ films on $\mathrm{LaAlO}_{3}$ is fully suppressed, whereas the metal-insulator transition temperature is considerably lowered in the $\mathrm{NdNiO}_{3}$ films on $\mathrm{LaAlO}_{3}$.
\end{abstract}

\title{
PACS: 71.30.+h
}

Key words: Nickel oxide, Metal-insulator transition, Epitaxial strain, Metal organic decomposition

*) Corresponding author. Address: Department of Applied Physics, Tokyo University of Agriculture and Technology, Naka-cho 2-24-16, Koganei, Tokyo 184-8588, Japan. Tel. +81 42388 7229; fax: +81 42385 6255. e-mail address: minaito@cc.tuat.ac.jp 


\section{Introduction}

The metal-insulator (M-I) transition triggered by strong Coulomb correlation $(U)$ in the transition-metal perovskite-related oxides have attracted a considerable interest over the last two decades since the discovery of high- $T_{\mathrm{c}}$ superconductivity in cuprates. The family of the rare-earth nickel oxides, $R E \mathrm{NiO}_{3}$, are one of the best candidates to study the M-I transition since the transition temperature varies systematically with the ionic size of $R E$. Furthermore, the M-I transition is not accompanied by any change in lattice symmetry, unlike the widely studied $\mathrm{V}_{2} \mathrm{O}_{3}$ or $\mathrm{Ti}_{2} \mathrm{O}_{3}$ system, but only a slight expansion of the unit cell on cooling into the insulating state, which makes the interpretation of the mechanism simple [1,2]. According to the framework developed by Zaanen, Sawatzky, and Allen [3], there are two general types of $U$-triggered insulators possible: the Mott-Hubbard type and the charge transfer type. The $R E \mathrm{NiO}_{3}$ materials belong to a wider family of charge-transfer-gap compounds, having a $p$-like valence band and a $d$-like conduction band, as is the case for high- $T_{\mathrm{c}}$ cuprates [4]. In fact, $R E \mathrm{NiO}_{3}$ are located at the boundary that separates "low charge-transfer gap metals" from "charge-transfer insulators" and the charge transfer gap is regulated by the ionic size of $R E^{3+}$ with a larger gap for smaller $R E^{3+}$ [5]. $\mathrm{LaNiO}_{3}$ is metallic with an enhanced Pauli paramagnetism [6] whereas, $\mathrm{LuNiO}_{3}$ is insulating with an antiferromagnetic ordering at $T_{\mathrm{N}}=$ $145 \mathrm{~K}$. $R E \mathrm{NiO}_{3}$ with $R E=$ Pr to Eu show a M-I transition and the M-I transition temperature $\left(T_{\mathrm{MI}}\right)$ increase from $135 \mathrm{~K}$ for $\mathrm{PrNiO}_{3}$ to $480 \mathrm{~K}$ for $\mathrm{EuNiO}_{3}[1,7]$. For smaller $R E^{3+}$, the perovskite tolerance factor $t$, where $t$ is defined as $t=d_{\mathrm{RE}-\mathrm{O}} / \sqrt{2} d_{\mathrm{Ni}-\mathrm{O}}$, is substantially smaller than $1.00(t=0.988$ for La versus $t=0.947$ for Ho, where $t$, which is evaluated for 12 -fold coordinated $R E^{3+}$, is taken from ref.1), which results in the severe distortion of the structure. In fact, $R E \mathrm{NiO}_{3}$ except for $R E=\mathrm{La}$ adopts the $\mathrm{GdFeO}_{3}$ structure, orthorhombically distorted perovskite structure $(\mathrm{Pbnm})$ with unit cell $\sim \sqrt{2} a_{\mathrm{p}} \times \sim \sqrt{2} a_{\mathrm{p}} \times \sim 2 a_{\mathrm{p}}$ ( $a_{\mathrm{p}}$ : the pseudo-cubic lattice parameter) at room temperature [1]. The most important feature of this distortion is 
the tilting of the $\mathrm{NiO}_{6}$ octahedra and resultant bending of the Ni-O-Ni bond. The Ni-O-Ni bond angle, $\theta$, deviates from the ideal $180^{\circ}$ to $165.2^{\circ}$ for La and $151.7^{\circ}$ for Ho [5]. With a decrease in $\theta$, the transfer matrix element between Ni3d and $\mathrm{O} 2 p$ orbitals is reduced, which makes both of Ni3d and $\mathrm{O} 2 p$ bands narrow, resulting in the opening of the charge-transfer gap.

The $T_{\mathrm{MI}}$ in $R E \mathrm{NiO}_{3}$ is also sensitive to applied static pressure as demonstrated by Canfield et al. [8] and Obradors et al. [9]. One would imagine that the application of physical pressure might cause the same effect as chemical pressure, i.e., reducing the size of $R E^{3+}$. But in fact, the charge transfer gap is reduced by physical pressure whereas it is enhanced by chemical pressure. This is because the tilting of the $\mathrm{NiO}_{6}$ octahedra arises from a mismatch of the $R E^{3+}$ size and the space between the corner-sharing octahedra: $R E^{3+}$ too small to fill this space causes $\mathrm{NiO}_{6}$ octahedra to tilt in order to make the space smaller. Pressure reduces the size of the space and consequently reduces the mismatch, leading to the decrease of the $\mathrm{NiO}_{6}$ tilting and the straightening of the Ni-O-Ni bond. Strain can also be induced in films by the lattice mismatch with substrates (so-called epitaxial strain). Recently there have been several articles reporting that the $T_{\mathrm{MI}}$ of $R E \mathrm{NiO}_{3}$ films shows a large shift by epitaxial strain, either compressive or tensile in-plane $[10,11,12]$. The stress in films is not isotropic but biaxial and thereby the out-of-plane strain is usually opposite to the in-plane strain due the Poisson effect, e.g. films compressed in-plane are stretched in the direction perpendicular to the film surface, and vice versa. This may cause a complex effect on the M-I transition and may be responsible for the variations among reported results.

As mentioned earlier, the $R E \mathrm{NiO}_{3}$ family is an ideal system for studying the M-I transition due to the simplicity of the materials and the proposed mechanism responsible for the M-I transition. But there is one problem, i.e., the nickel ions in $R E \mathrm{NiO}_{3}$ exist in the +3 oxidation state. Since this oxidation state is unstable in ambient oxygen pressure, the synthesis of the $R E \mathrm{NiO}_{3}$ materials typically requires either processing at high oxygen 
pressures or at low temperatures to stabilize $\mathrm{Ni}^{3+}$. The difficulty in the synthesis of $R E \mathrm{NiO}_{3}$ increases markedly as the $R E^{3+}$ ionic size decreases. However, the intensive efforts over the last decade, have demonstrated that thin film synthesis of $R E \mathrm{NiO}_{3}$ is not as difficult as bulk synthesis due to low-temperature processing and also possibly due to the virtue of epitaxial stabilization. Furthermore single-crystalline films can be obtained, which makes this avenue even more attractive. Most of the past efforts to synthesize $R E \mathrm{NiO}_{3}$ films except for $R E=$ La have been performed by either pulsed laser deposition (PLD) [13] or RF magnetron sputtering [10]. In this article, we demonstrate the synthesis of single-crystalline $R E \mathrm{NiO}_{3}$ thin films $(R E=\mathrm{La}, \mathrm{Pr}, \mathrm{Nd}, \mathrm{Sm}$, and $\mathrm{Eu})$ by metal organic decomposition (MOD) via solid-state epitaxy. Although MOD is fundamentally not different from a solid-state reaction, it appears to stabilize the $\mathrm{Ni}^{3+}$ valence state without the need for high oxygen pressure even for smaller $R E^{3+}$ ions. The films prepared by MOD show high crystallinity and low resistivity. The resistivity of $\mathrm{LaNiO}_{3}$ at $300 \mathrm{~K}$ is as low as $100 \mu \Omega \mathrm{cm}$. Furthermore we have investigated the influence of epitaxial strain on the film characteristics and observed a dramatic effect of in-plane compressive strain on the $\mathrm{M}$-I transition in $\mathrm{PrNiO}_{3}$ and $\mathrm{NdNiO}_{3}$ films on $\mathrm{LaAlO}_{3}$ substrates.

\section{Experimental}

\section{1. Thin film synthesis}

$R E \mathrm{NiO}_{3}$ films were prepared by MOD using 2-ethylhexanoate solutions for La and $\mathrm{Ni}$ and naphthenate solutions for $\mathrm{Pr}, \mathrm{Nd}, \mathrm{Sm}$, and $\mathrm{Eu}$. The stoichiometric mixture of solutions was spin-coated on $\mathrm{LaAlO}_{3}$ (LAO) (001), $\mathrm{NdGaO}_{3}$ (NGO) (110), and $\mathrm{SrTiO}_{3}$ (STO) (001) substrates. The lattice constants of the substrates, $a_{\mathrm{s}}$, are summarized in Table I, together with the lattice constants reported for bulk samples of $R E \mathrm{NiO}_{3}$. The films were first calcined at $500^{\circ} \mathrm{C}$ in air to obtain precursors, then fired at $650-900^{\circ} \mathrm{C}$ in a tubular furnace with flowing either molecular oxygen or ozone gas from a commercial ozonizer $(\leq 5 \%$ ozone 
concentration). Subsequently the films were furnace-cooled down to $300{ }^{\circ} \mathrm{C}$. The film thickness was typically $800 \AA$ although it may vary from film to film to some extent. The structure of the films was characterized by $\omega-2 \theta$ (equivalently $\theta-2 \theta$ ) scans with $\mathrm{Cu} \mathrm{K}_{\alpha}$ radiation using Rigaku, Smart Lab. From the $\omega-2 \theta$ scans, the crystallinity was assessed by the peak intensity as well as the rocking curve width $(\Delta \omega)$, and also the out-of-plane lattice constant, namely perpendicular to the film surface (hereafter referred as $c_{\mathrm{f}}$ ), was determined. Some of the films with high crystallinity were also examined by the four-circle $(2 \theta, \omega, \phi, \psi)$ X-ray diffractometer (Phillips, X'Pert). The in-plane alignment was determined by the $\phi$ scan of the (103) and/or (202) reflections and its width $(\Delta \phi)$. Also, the in-plane lattice constant (hereafter referred as $a_{\mathrm{f}}$ ) was determined from the (110) reflection [14]. The resistivity of the films was measured by a standard four-probe method.

\section{2. Optimizing the growth parameters}

First of all, the growth parameters (firing temperature and atmosphere $\left(\mathrm{O}_{2}\right.$ or $\left.\mathrm{O}_{3}\right)$ ) for the synthesis of $R E \mathrm{NiO}_{3}$ were optimized for each $R E$. Figure 1 summarizes the optimization process. The XRD peak intensity of the (002) reflection (Fig. 1 (a)) and the resistivity at room temperature, $\rho(295 \mathrm{~K})\left(\right.$ Fig. 1 (b)), are plotted as a function of the firing temperature, $T_{\mathrm{f}}$, for typical $R E \mathrm{NiO}_{3}$ films on $\mathrm{NGO}$ substrates prepared under either $\mathrm{O}_{2}$ or $\mathrm{O}_{3}$. For $R E=\mathrm{La}$, the $\mathrm{O}_{2}$ growth and the $\mathrm{O}_{3}$ growth are not much different and hence the films were prepared mostly under $\mathrm{O}_{2}$. For the film preparation under $\mathrm{O}_{2}$, the (002) peak intensity increases with $T_{\mathrm{f}}$ from $650^{\circ} \mathrm{C}$ to $900^{\circ} \mathrm{C}$ whereas the $\rho(295 \mathrm{~K})$ decreases gradually with increase in $T_{\mathrm{f}}$. For $R E=\operatorname{Pr}$ and $\mathrm{Nd}$, the film growth under $\mathrm{O}_{3}$ is slightly better than that under $\mathrm{O}_{2}$. For $\mathrm{PrNiO}_{3}$

films prepared under $\mathrm{O}_{3}$, the film quality improves monotonically with $T_{\mathrm{f}}$ from $650^{\circ} \mathrm{C}$ to $800^{\circ} \mathrm{C}$ as evident from the increase in the (002) peak intensity and the decrease in $\rho(295 \mathrm{~K})$. However, the $\rho(295 \mathrm{~K})$ increases for $T_{\mathrm{f}} \geq 850^{\circ} \mathrm{C}$. For $R E=\mathrm{Nd}$, although the data are scattered, the optimum $T_{\mathrm{f}}$ appears to be lower than for $R E=\operatorname{Pr}$. The optimization for $\mathrm{PrNiO}_{3}$ 
and $\mathrm{NdNiO}_{3}$ has been carried out not only from a viewpoint of the XRD peak intensity and $\rho(295 \mathrm{~K})$, but also from the sharpness of the M-I transition. Figure 2 compares the M-I transition for $\mathrm{PrNiO}_{3}$ films on $\mathrm{NGO}$ fired at various $T_{\mathrm{f}}$ under $\mathrm{O}_{3}$ flow. One can see an overall tendency that lower $T_{\mathrm{f}}$ yields a sharper M-I transition.

For $R E=\mathrm{Sm}$ and $\mathrm{Eu}$, the films were prepared mostly with $\mathrm{O}_{3}$. Even with $\mathrm{O}_{3}$, $\mathrm{SmNiO}_{3}$ films prepared with $T_{\mathrm{f}}=900^{\circ} \mathrm{C}$ show broad XRD peaks with the appearance of an impurity phase, $\mathrm{Sm}_{2} \mathrm{O}_{3}$, and also have high resistivity values. For $R E=\mathrm{Eu}$, firing at $T_{\mathrm{f}} \geq$ $850^{\circ} \mathrm{C}$ yields mainly $\mathrm{Eu}_{2} \mathrm{O}_{3}$. The optimum synthesis conditions are summarized in Table II. The $R E$ dependence of the synthesis conditions for $R E \mathrm{NiO}_{3}$ seems to indicate that the thermodynamic boundary conditions become more stringent for smaller $R E^{3+}$ ions, indicating that stronger oxidation and/or lower firing temperature are required. But in general, the reaction activity is lower for smaller $R E^{3+}$, which makes it difficult to promote crystal growth at low $T_{\mathrm{f}}$.

\section{Results and discussion}

\section{1. $\mathrm{RENiO}$ films on $\mathrm{NdGaO}_{3}$ substrates}

First, we discuss our results of $R E \mathrm{NiO}_{3}$ films on $\mathrm{NdGaO}_{3}$ substrates. $\mathrm{NdGaO}_{3}$ is nearly lattice-matched with $R E \mathrm{NiO}_{3}(R E=\mathrm{La}, \mathrm{Pr}, \mathrm{Nd})$, and most frequently used substrate for thin film growth of these compounds. Taking a closer look at the lattice constants as shown in Table $\mathrm{I}$, it is clear that the $a_{\mathrm{s}}$ of $\mathrm{NdGaO}_{3}$ is a little larger than the pseudo-cubic perovskite lattice constant, $a_{\mathrm{p}}$ (bulk), of $R E \mathrm{NiO}_{3}$, which should introduce in-plane tensile strain into $R E \mathrm{NiO}_{3}$ films. Figure 3 shows the out-of-plane XRD patterns (a) and resistivity-temperature $(\rho-T)$ curves (b) of $R E \mathrm{NiO}_{3}$ films with $R E=\mathrm{La}, \mathrm{Pr}, \mathrm{Nd}, \mathrm{Sm}$, and $\mathrm{Eu}$, synthesized under optimized conditions. All the peaks in the XRD patterns except for the substrate peaks could be indexed to the $(00 l)$ reflections of the $R E \mathrm{NiO}_{3}$ perovskite structure, indicating that the films are single-phase and $c$-axis textured. However, the peak intensity becomes weaker and 
broader with decrease in the $R E^{3+}$ ionic radius, i.e., going from La to Eu. Figure 4 summarizes the variation $c_{\mathrm{f}}$ of the films with the ionic radius of 12 -fold coordinated $R E^{3+}$. The $c_{\mathrm{f}}$ of the films on $\mathrm{NdGaO}_{3}$ for $R E=\mathrm{La}, \mathrm{Pr}$, and $\mathrm{Nd}$ are smaller than the $a_{\mathrm{p}}$ (bulk) reported for bulk specimens, implying that an in-plane tensile strain is induced in these films. In order to confirm this speculation, the in-plane structural characterizations were carried out using the four-circle X-ray diffractometer. Table III presents a summary of all the structural data. Figure 5 demonstrates the $\phi$ scan of the (103) reflection for the $\mathrm{PrNiO}_{3}$ film on $\mathrm{NdGaO}_{3}$ as a typical example. The four-fold symmetry observed in the $\phi$-scan XRD pattern of the films indicates that the films are indeed in-plane aligned. The full width at half maximum, $\Delta \phi$, is $0.66^{\circ}$, a fairly good value for complex oxide thin films. Furthermore, the in-plane lattice constant, $a_{\mathrm{f}}$, of the films was evaluated from the $\omega-2 \theta$ scan of the (110) reflection. As shown in Table III, the $a_{\mathrm{f}}$ of the films on $\mathrm{NdGaO}_{3}$ for $R E=\mathrm{La}, \mathrm{Pr}$, and $\mathrm{Nd}$ estimated from the maximum of the peak are indeed larger than the $a_{\mathrm{p}}(\mathrm{bulk})$, confirming the in-plane tensile strain induced in these films by solid-state epitaxy. However, the $\omega-2 \theta$ scan of the (110) reflection for the $\mathrm{PrNiO}_{3}$ films on $\mathrm{NGO}$ is fairly broad (Fig. 6(a)) indicating that there is a substantial variation of the $a_{\mathrm{f}}$ in the film, probably along the thickness direction. The $a_{\mathrm{f}}$ may be close to $a_{\mathrm{s}}$ near the interface between a film and a substrate, but the strain is relaxed toward the film surface. Therefore $\Delta a_{\mathrm{f}}$, calculated from the full width $(\Delta(2 \theta))$ at the half maximum for the (110) reflection, is included in Table III.

The resistivity value above the $T_{\mathrm{MI}}$ increases monotonically from $\mathrm{La}$ to $\mathrm{Nd}$ as shown in Fig. 3(b). This trend in the resistivity is partly due to the poorer crystallinity and predominantly due to the larger bending of the Ni-O-Ni bond for smaller $R E^{3+} . \quad \mathrm{LaNiO}_{3}$ film exhibits low resistivity, $\rho(295 \mathrm{~K})=100 \mu \Omega \mathrm{cm}$, and a high residual resistivity ratio, $R R R$ (三 $\rho(295 \mathrm{~K}) / \rho(4.2 \mathrm{~K}))$, of $\sim 18$, both of which are comparable to or even better than the best values ever reported $[8,15] . \quad \mathrm{PrNiO}_{3}$ and $\mathrm{NdNiO}_{3}$ films show a $\mathrm{M}-\mathrm{I}$ transition at $125 \mathrm{~K}$ and $210 \mathrm{~K}$ respectively, which roughly agree with the $T_{\mathrm{MI}}$ reported for the bulk polycrystalline 
ceramics $\left(T_{\mathrm{MI}}=130-135 \mathrm{~K}\right.$ for $\mathrm{Pr}$, and $T_{\mathrm{MI}}=200 \mathrm{~K}$ for $\left.\mathrm{Nd}\right)[1,16]$. According to the previous reports $[1,5,7]$, the $T_{\mathrm{MI}}$ 's of $\mathrm{SmNiO}_{3}$ and $\mathrm{EuNiO}_{3}$ are $400 \mathrm{~K}$ and $480 \mathrm{~K}$, respectively. Therefore we observed only a semiconducting temperature dependence of resistivity below the M-I transition for these two compounds in the measured temperature range $(300-4.2 \mathrm{~K})$.

The $T_{\mathrm{MI}}$ of $R E \mathrm{NiO}_{3}$ films is affected by several factors such as epitaxial strain, crystallinity, etc. In-plane compressive strain reduces the $T_{\mathrm{MI}}$ whereas tensile strain raises it. Poor crystallinity reduces the $T_{\mathrm{MI}}$ and broadens the transition. Our $T_{\mathrm{MI}}(=210 \mathrm{~K})$ for $\mathrm{NdNiO}_{3}$ may be slightly higher but our $T_{\mathrm{MI}}(=125 \mathrm{~K})$ for $\mathrm{PrNiO}_{3}$ is slightly lower than the bulk $T_{\mathrm{MI}}$ reported for these compounds. It has been reported that the $\mathrm{NdNiO}_{3}$ films as thin as $200 \AA$ prepared by PLD on NGO show $T_{\mathrm{MI}}=240 \mathrm{~K}$ [12], but this is an exceptional case as the film thickness is very low. In our observations, the increase of $T_{\mathrm{MI}}$ by tensile strain was not that prominent.

As mentioned in introduction section, $R E \mathrm{NiO}_{3}$ except for $R E=\mathrm{La}$ adopts the orthorhombic $\mathrm{GdFeO}_{3}$ structure. But another form of $\mathrm{NdNiO}_{3}$ with a rhombohedral unit cell has been synthesized by low-temperature $\left(\sim 650^{\circ} \mathrm{C}\right)$ bulk synthesis at $p_{\mathrm{O} 2}=1$ atm [17]. This phase has a somewhat larger lattice constant $\left(a_{\mathrm{p}}(\mathrm{bulk})=3.85 \AA\right.$ for the pseudo-cubic cell), and shows a much more broader M-I transition with a much larger transition hysteresis on cooling and heating $\left(\Delta T_{\mathrm{MI}} \sim 100 \mathrm{~K}\right)$ than orthorhombic $\mathrm{NdNiO}_{3}\left(a_{\mathrm{p}}(\right.$ bulk $\left.)=3.81 \AA, \Delta T_{\mathrm{MI}} \leq 8-12 \mathrm{~K}\right)[8$, 18]. It should be noted that our MOD preparation conditions for $\mathrm{NdNiO}_{3}$ films are almost identical to the bulk synthesis conditions for rhombohedral $\mathrm{NdNiO}_{3}$. However, a sharp M-I transition and a small hysteresis $\left(\Delta T_{\mathrm{MI}} \leq 10 \mathrm{~K}\right)$ observed in our $\mathrm{NdNiO}_{3}$ films (Fig. 7) seems to indicate that our $\mathrm{NdNiO}_{3}$ films may be orthorhombic $\mathrm{NdNiO}_{3}$. The bulk synthesis of orthorhombic $\mathrm{NdNiO}_{3}$ requires oxygen pressures of 150-200 bars [1] whereas its MOD synthesis requires only 1 atm of oxygen [19], which is remarkable. This large difference in oxygen pressure during synthesis to stabilize orthorhombic $\mathrm{NdNiO}_{3}$ is puzzling.

It is worthwhile to point out that the $c_{\mathrm{f}}$ values for $\mathrm{SmNiO}_{3}$ and $\mathrm{EuNiO}_{3}$ films on $\mathrm{NGO}$ 
are longer than the $\mathrm{NdNiO}_{3}$ films on $\mathrm{NGO}$ (please see Fig. 4) and also longer than the $a_{\mathrm{p}}$ (bulk) by $\sim 0.5 \%$. This cannot be explained by epitaxial strain. The longer $c_{\mathrm{f}}$ values observed for $R E=\mathrm{Sm}$ and Eu might be attributed to rhombohedral distortion as in the case of rhombohedral $\mathrm{NdNiO}_{3}$. Our oxidation condition, even with $\mathrm{O}_{3}$, during synthesis may not be strong enough to stabilize the orthorhombic phase of $\mathrm{SmNiO}_{3}$ and $\mathrm{EuNiO}_{3}$.

\section{2. $R E \mathrm{NiO}_{3}$ films on $\mathrm{LaAlO}_{3}$ substrates}

We now examine our results for the films on LAO. LAO has smaller $a_{\mathrm{s}}$ than $a_{\mathrm{p}}\left(\right.$ bulk) of $R E \mathrm{NiO}_{3}(R E=\mathrm{La}, \mathrm{Pr}, \mathrm{Nd})$, so it should introduce in-plane compressive strain into $R E \mathrm{NiO}_{3}$ films. Indeed, there are several recent articles demonstrating a large epitaxial strain effect on the M-I transition in the $\mathrm{PrNiO}_{3}$ and $\mathrm{NdNiO}_{3}$ films on $\mathrm{LAO}[11,20]$. The structural data in Fig. 4 and Table III show that our MOD-grown $\mathrm{PrNiO}_{3}$ and $\mathrm{NdNiO}_{3}$ films on $\mathrm{LAO}$ have smaller $a_{\mathrm{f}}$ and larger $c_{\mathrm{f}}$ compared to their respective $a_{\mathrm{p}}(\mathrm{bulk})$, indicating that these films are well strained via solid-state epitaxy. As shown in Fig. 6(b), the peak width of the (110) reflection in the $\omega-2 \theta$ scan for the $\mathrm{PrNiO}_{3}$ film on LAO is relatively small as compared to the $\mathrm{PrNiO}_{3}$ film on NGO (Fig. 6(a)) or on STO (Fig. 6(c)). The peak is asymmetric with a tail at lower angles, implying the existence of portions with larger $a_{\mathrm{f}}$ in the film due to probable strain relaxation.

Figure 8 compares the $\rho$-T curves of $R E \mathrm{NiO}_{3}$ films for $R E=\mathrm{La}, \mathrm{Pr}, \mathrm{Nd}$, and $\mathrm{Sm}$ prepared on $\mathrm{LAO}, \mathrm{NGO}$ and $\mathrm{STO}$ substrates. For $R E=\mathrm{La}$, the substrate dependence is minimal, but a better lattice-matched NGO gives the lowest resistivity and highest $R R R$ values. On the other hand, for $R E=\operatorname{Pr}$, the substrate dependence of the resistivity is dramatic. The M-I transition of the $\mathrm{PrNiO}_{3}$ films on LAO is completely suppressed and the film shows a $R R R$ value as high as 15 . In the case of $R E=\mathrm{Nd}$, the $T_{\mathrm{MI}}$ is also reduced substantially from $210 \mathrm{~K}$ to $140 \mathrm{~K}$ although the transition is even sharper for the films on LAO than on NGO. For $R E=\mathrm{Sm}$, again the substrate dependence is small. The reduction of $T_{\mathrm{MI}}$ in $\mathrm{PrNiO}_{3}$ and 
$\mathrm{NdNiO}_{3}$ is roughly equal to the reduction by static isotropic pressure of $\sim 15 \mathrm{kbar}$.

\section{3. $\mathrm{RENiO}$ films on $\mathrm{SrTiO}_{3}$ substrates}

STO has been the most common substrate for oxide film growth. It has substantially larger $a_{\mathrm{s}}$ than $a_{\mathrm{p}}\left(\right.$ bulk) of $R E \mathrm{NiO}_{3}$, which should introduce in-plane tensile epitaxial strain into $R E \mathrm{NiO}_{3}$ films. Since the lattice mismatch of $R E \mathrm{NiO}_{3}$ with $\mathrm{STO}$ is larger than that of NGO, a more dramatic epitaxial strain effect would be expected. However, the structural data in Fig. 4 and Table III, show that the situation is not that straightforward. The $c_{\mathrm{f}}$ should be reduced by the in-plane tensile strain via the Poisson effect. The $c_{\mathrm{f}}$ of $\mathrm{LaNiO}_{3}$ is indeed smaller on STO than on NGO. In contrast, the $c_{\mathrm{f}}$ of $\mathrm{PrNiO}_{3}$ and $\mathrm{NdNiO}_{3}$ is larger on STO than on NGO contrary to the expectation. Furthermore, the $a_{\mathrm{f}}$ of $\mathrm{PrNiO}_{3}$ is larger on STO than on NGO as expected whereas the $a_{\mathrm{f}}$ of $\mathrm{LaNiO}_{3}$ and $\mathrm{NdNiO}_{3}$ is smaller on $\mathrm{STO}$ than on NGO as opposed to expectation. This complex behavior seems to imply that the epitaxial strain is relaxed, at least partially, on STO due to too large lattice mismatch. This scenario is supported by the $\omega-2 \theta$ scan (Fig. 6(c)) for the $\mathrm{PrNiO}_{3}$ film on $\mathrm{STO}$ where the peak width of the (110) reflection on STO is considerably larger than on LAO or NGO.

In line with the structural observations, the $\rho$ - $T$ curves show an unusual behavior. The in-plane tensile strain is expected to raise the $T_{\mathrm{MI}}$. Contrary to this expectation, the $T_{\mathrm{MI}}$ of the $\mathrm{PrNiO}_{3}$ films on $\mathrm{STO}$ is reduced from $130 \mathrm{~K}$ to $\sim 100 \mathrm{~K}$ and the transition is also substantially broadened. This tendency is more prominent in the case of $\mathrm{NdNiO}_{3}$ films on STO where the M-I transition is quite smeared. Because of this smearing, the $T_{\mathrm{MI}}$ is difficult to determine precisely but seems to be lower than $T_{\mathrm{MI}}$ of the films on NGO. In general, the critical thickness for the relaxation of epitaxial strain is inversely proportional to the lattice mismatch $\alpha\left(=\Delta a / a_{\mathrm{p}}(\right.$ bulk $\left.)\right)$ where $\Delta a=a_{\mathrm{s}}-a_{\mathrm{p}}($ bulk $) . \quad$ The value of $\alpha$ is listed in Table III. For $\mathrm{PrNiO}_{3}$, the $\alpha$ is $+2.36 \%$ for STO, $+1.17 \%$ for NGO, and $-0.45 \%$ for LAO. The film

thickness of about $800 \AA$ in the present study may be under the critical thickness for LAO but 
over the critical thickness for STO, with NGO on the border. The $\alpha$ of STO is $+2.36 \%$ for $\mathrm{PrNiO}_{3}$ and $+2.56 \%$ for $\mathrm{NdNiO}_{3}$. The larger $\alpha$ may explain that $\mathrm{NdNiO}_{3}$ films on $\mathrm{STO}$ show a more smeared M-I transition than $\mathrm{PrNiO}_{3}$ films on STO.

\subsection{Comparison of $\mathrm{RENiO}_{3}$ films by MOD with films by other thin film techniques}

Now we would like to make a comparison of the properties of our MOD films with those grown by other thin film techniques such as MBE [21,24], PLD [13,12,22,11,26], RF magnetron sputtering [23,27], and MOCVD [25], which is summarized in Table IV [28]. In the literature, most of the earlier works on the growth of $\mathrm{LaNiO}_{3}$ films were by MBE, PLD, and sputtering, and MBE and PLD grown films have high $\rho(295 \mathrm{~K})$ and low $R R R$ values. Only the $\mathrm{LaNiO}_{3}$ films prepared by sputtering have $\rho(295 \mathrm{~K})$ and $R R R$ values comparable to our MOD synthesized films.

The $\mathrm{PrNiO}_{3}$ films have been grown by MBE, PLD, and MOCVD. The M-I transition is fully suppressed in the well-strained films grown on LAO either by MBE or PLD whereas only slight reduction of $T_{\mathrm{MI}}$ is seen in the MOCVD grown films, which may be due to partial strain-relaxation. In spite of full suppression of the M-I transition in MBE and PLD grown films, the films have lower $R R R$ than our MOD grown films $(2 \sim 4$ in MBE or PLD versus 15 in MOD), indicating that $\mathrm{PrNiO}_{3}$ films by MOD may be superior to those by MBE or PLD.

The $\mathrm{NdNiO}_{3}$ films have been grown by PLD and sputtering. On LAO substrates, the films shows the reduction of $T_{\mathrm{MI}}$ due to in-plane compressive strain although $T_{\mathrm{MI}}$ ranges from $80 \mathrm{~K}$ to $140 \mathrm{~K}$, depending on several factors such as thickness, crystallinity, etc. On NGO substrates, the increase of $T_{\mathrm{MI}}$ to $240 \mathrm{~K}$ was observed due to in-plane tensile strain in very thin (200 $\AA)$ films grown by PLD, but this is rather exceptional. In most other reports, the $T_{\mathrm{MI}}$ on NGO is reduced to $150-170 \mathrm{~K}$, possibly due to poor crystallinity, which is known to reduce the $T_{\mathrm{MI}}$ and broaden the M-I transition, as mentioned earlier. Judging from this 
criterion, our $\mathrm{NdNiO}_{3}$ films $\left(T_{\mathrm{MI}}=210 \mathrm{~K}\right)$ on $\mathrm{NGO}$ are fair, at least. Taking into account the simplicity and low cost of MOD, it is a quite attractive method to synthesize high-quality $R E \mathrm{NiO}_{3}$ thin films. We have also demonstrated that these MOD films are suitable as precursors for topotactic transformation to infinite-layer $R E \mathrm{NiO}_{2}[29]$.

\section{Summary}

We have carried out the systematic synthesis of $R E \mathrm{NiO}_{3}$ films $(R E=\mathrm{La}, \mathrm{Pr}, \mathrm{Nd}, \mathrm{Sm}$, and $\mathrm{Eu}$ ) by MOD on NGO, LAO, and STO substrates and the influence of epitaxial strain on various properties was investigated from the structural characterization and transport measurements. The results are summarized as follows.

(1) $\mathrm{RENiO}_{3}$ films on $\mathrm{NGO}$ - in-plane tensile strain

The $\mathrm{LaNiO}_{3}$ films on NGO show high crystallinity due to large $\mathrm{La}^{3+}$ as well as good lattice matching with the substrate. The best film exhibits $\rho(295 \mathrm{~K})=100 \mu \Omega \mathrm{cm}$, and a large $R R R$ value of $\sim 18$. The $\mathrm{PrNiO}_{3}$ and $\mathrm{NdNiO}_{3}$ films on $\mathrm{NGO}$ have slight in-plane tensile strain, and they show a M-I transition at $125 \mathrm{~K}$ and $210 \mathrm{~K}$ respectively. The $T_{\mathrm{MI}}=210 \mathrm{~K}$ for $\mathrm{NdNiO}_{3}$ films prepared by MOD is slightly higher than the $T_{\mathrm{MI}}$ reported for bulk samples, which could be attributed to tensile strain.

(2) $R E \mathrm{NiO}_{3}$ films on $\mathrm{LAO}$ - in-plane compressive strain

The $\mathrm{PrNiO}_{3}$ and $\mathrm{NdNiO}_{3}$ films on $\mathrm{LAO}$ experience in-plane compressive strain. The structural characterization of the $\mathrm{MOD}$-grown $\mathrm{PrNiO}_{3}$ and $\mathrm{NdNiO}_{3}$ films revealed that the films are well strained on LAO via solid-state epitaxy. The M-I transition of the $\mathrm{PrNiO}_{3}$ films on LAO is fully suppressed and the films are metallic down to $4.2 \mathrm{~K}$ with a $R R R$ value as high as 15 . The $T_{\mathrm{MI}}$ of the $\mathrm{NdNiO}_{3}$ films is also reduced significantly from $210 \mathrm{~K}$ to 140 $\mathrm{K}$ besides having a sharp transition.

(3) $\mathrm{RENiO}_{3}$ films on $\mathrm{STO}$ - strain relaxation

In-plane tensile strain is introduced into the $\mathrm{RENiO}_{3}$ films but is partially relaxed due to too 
large lattice mismatch. The $T_{\mathrm{MI}}$ of the $\mathrm{PrNiO}_{3}$ films on $\mathrm{STO}$ is reduced from $130 \mathrm{~K}$ to $\sim 100$ $\mathrm{K}$ with the transition substantially broadened. This effect is more prominent in the $\mathrm{NdNiO}_{3}$ films on STO and the M-I transition is quite smeared.

Progress in the methods of computational material science may now allow first-principle calculations of the coupling between structure and electronic properties. Some attempts have started toward understanding the epitaxial strain effect on the electronic structure in $R E \mathrm{NiO}_{3}$, although still rather crude [30,31]. It will require state-of-the-art computational routes to explain the dramatic changes in the physical properties caused by a change in the lattice constants as small as $\pm 1 \%$.

\section{Acknowledgements}

The work was partly supported by KAKENHI B (23340098) from Japan Society for the Promotion of Science (JSPS) and also by Advanced Low Carbon Technology (ALCA), Japan Science and Technology Agency. The authors thank J. Kurian, Darmstadt University of Technology, for critical reading of the manuscript. 


\section{References}

[1] P. Lacorre, J. B. Torrance, J. Pannetier, A. I. Nazzal, P. W. Wang, and T. C. Huang, J. Solid State Chem. 91, 225 (1991).

[2] J. L. García-Muñoz, J. Rodríguez-Carvajal, P. Lacorre, and J. B. Torrance, Phys. Rev. B 46 (8), 4414 (1992).

[3] J. Zaanen, G. A. Sawatzky, and J. W. Allen, Phys. Rev. Lett. 55, 418 (1985).

[4] J. B. Torrance, P. Lacorre, C. Asavaroengchai, and R. M. Metzger, Physica C 182, $351(1991)$.

[5] J. B. Torrance, P. Lacorre, A. I. Nazzal, E. J. Ansaldo, and Ch. Niedermayer, Phys. Rev. B 45 (14), 8209 (1992).

[6] J. B. Goodenough and P. M. Raccah, J. Appl. Phys. 36 (3), 1031 (1965).

[7] J. A. Alonso, M. J. Martínez-Lope, and I. Rasines, J. Solid State Chem. 120, 170 (1995).

[8] P. C. Canfield, J. D. Thompson, S-W. Cheong, and L. W. Rupp, Phys. Rev. B 47 (18), 12357 (1993).

[9] X. Obradors, L. M. Paulius, M. B. Maple, J. B. Torrance, A. I. Nazzal, J. Fontcuberta, and X. Granados, Phys. Rev. B 47 (18), 12353 (1993).

[10] J. F. DeNatale, and P. H. Korbin, J. Mater. Res. 10 (12), 2992 (1995).

[11] A. Venimadhav, I. C. Lekshmi, and M. S. Hegde, Materials Research Bulletin 37, 201 (2002).

[12] R. Eguchi, Y. Okamoto, Z. Hiroi, S. Shin, A. Chainani, Y. Tanaka, M. Matsunami, Y. Takata, Y. Nishino, K. Tamasaku, M. Yabashi, and T. Ishikawa, J. Appl. Phys. 105, 056103 (2009).

[13] G. Catalan, R. M. Bowman, and J. M. Gregg, Phys. Rev. B 62 (12), 7892 (2000).

[14] It was not possible to determine $a_{\mathrm{f}}$ using the (200) reflection of the film due to the heavy interference by the strong substrate reflection. 
[15] J.-S. Zhou, J. B. Goodenough, and B. Dabrowski, Phys. Rev. B 67, 020404 (2003).

[16] We define $T_{\mathrm{MI}}$ in this article the temperature where the resistivity starts to rise.

[17] J. K. Vassiliou, M. Hornbostel, R. Ziebarth, and F. J. Disalvo, J. Solid State Chem. 81, 208 (1989).

[18] X. Granados, J. Fontcuberta, X. Obradors, LI. Mañosa, and J. B. Torrance, Phys. Rev. B, 48 (16), 11666 (1993).

[19] The phase forms with 1 atm of molecular oxygen although the growth with ozone is slightly better.

[20] J. Liu, M. Kareev, B. Gray, J. W. Kim, P. Ryan, B. Dabrowski, J. W. Freeland, and J. Chakhalian, Appl. Phys. Lett. 96, 233110 (2010).

[21] S. J. May, T. S. Santos, and A. Bhattacharya, Phys. Rev. B 79, 115127 (2009).

[22] M. Kawai, S. Inoue, M. Mizumaki, N. Kawamura, N. Ichikawa, and Y. Shimakawa, Appl. Phys. Lett. 94, 082102 (2009).

[23] J. Son, P. Moetakef, J. M. LeBeau, D. Ouellette, L. Balents, S. J. Allen, and S. Stemmer, Appl. Phys. Lett. 96, 062114 (2010).

[24] L. Feigl, B. D. Schultz, S. Ohya, D. G. Ouellette, A. Kozhanov, and C. J. Palmstrøm, J. Cryst. Growth 366, 51 (2013).

[25] M. A. Novojilov, O. Yu. Gorbenko, I. E. Graboy, A. R. Kaul, H. W. Zandbergen, N. A. Babushkina, and L. M. Belova, Appl. Phys. Lett. 76 (15), 2041 (2000).

[26] U. Staub, G. I. Meijer, F. Fauth, R. Allenspach, J. G. Bednorz, J. Karpinski, S. M. Kazakov, L. Paolasini, and F. d’ Acapito, Phy. Rev. Lett. 88 (12), 126402 (2002).

[27] R. Scherwitzl, P. Zubko, I. G. Lezama, S. Ono, A. F. Morpurgo, G. Catalan, and J-M. Triscone, Adv. Mater. 22, 5517 (2010).

[28] The data for ultrathin $(<100 \AA)$ films are excluded since they may have any possible surface effect such as reconstruction, oxygen loss, etc.

[29] A. Ikeda, T. Manabe, and M. Naito, Physica C 495, 134 (2013). 
[30] A. Yu. Dobin, K. R. Nikolaev, I. N. Krivorotov, R. M. Wentzcovitch, E. Dan Dahlberg, and A. M. Goldman, Phys. Rev. B 68, 113408, 2003.

[31] Sergey Prosandeev, L. Bellaiche, and Jorge Íñiguez, Phys. Rev. B 85, 214431, 2012. 
Table I. Crystal structure and the lattice parameters of $R E \mathrm{NiO}_{3}[1,7]$ and the substrate materials used in this work. The pseudo-cubic perovskite lattice constant, $a_{\mathrm{p}}(\mathrm{bulk})$, for $R E \mathrm{NiO}_{3}$ and, $a_{\mathrm{s}}$, for the substrates are calculated by assuming the materials as a pseudo-cubic system.

\begin{tabular}{cccccc}
\hline $\begin{array}{c}\text { Material or } \\
\text { substrate }\end{array}$ & Structure & $a(\AA)$ & $b(\AA)$ & $c(\AA)$ & $a_{\mathrm{p}}($ bulk $)$ or $a_{\mathrm{s}}(\AA)$ \\
\hline \hline $\mathrm{LaNiO}_{3}$ & rhombohedral & $\begin{array}{c}5.3954 \\
\left(\alpha=60^{\circ} 78^{\prime}\right)\end{array}$ & & 3.830 \\
$\mathrm{PrNiO}_{3}$ & $\mathrm{GdFeO}_{3}$ & 5.4154 & 5.3755 & 7.6192 & 3.813 \\
$\mathrm{NdNiO}_{3}$ & $\mathrm{GdFeO}_{3}$ & 5.3888 & 5.3845 & 7.6127 & 3.805 \\
$\mathrm{SmNiO}_{3}$ & $\mathrm{GdFeO}_{3}$ & 5.3283 & 5.4374 & 7.5675 & 3.782 \\
$\mathrm{EuNiO}_{3}$ & $\mathrm{GdFeO}_{3}$ & 5.2938 & 5.5460 & 7.5359 & 3.768 \\
$\mathrm{SrTiO}_{3}(001)$ & $\mathrm{Perovskite}$ & & & & 3.905 \\
$\mathrm{NdGaO}_{3}(110)$ & $\mathrm{GdFeO}_{3}$ & 5.417 & 5.499 & 7.717 & 3.858 \\
$\mathrm{LaAlO}_{3}(001)$ & rhombohedral & 5.356 & & & 3.796 \\
& & $\left(\alpha=60^{\circ} 06^{\prime}\right)$ & & \\
\hline
\end{tabular}


Table II. Summary of the optimal synthesis condition for $R E \mathrm{NiO}_{3}(R E=\mathrm{La}, \mathrm{Pr}, \mathrm{Nd}, \mathrm{Sm}$, and $\mathrm{Eu})$ films on NGO.

\begin{tabular}{ccc}
\hline$R E$ & $T_{\mathrm{f}}\left({ }^{\circ} \mathrm{C}\right)$ & $\mathrm{O}_{2}$ or $_{3}$ \\
\hline $\mathrm{La}$ & 850 & $\mathrm{O}_{2}$ \\
$\mathrm{Pr}$ & $700-800$ & $\mathrm{O}_{3}$ \\
$\mathrm{Nd}$ & $650-750$ & $\mathrm{O}_{3}$ \\
$\mathrm{Sm}$ & $750-850$ & $\mathrm{O}_{3}$ \\
$\mathrm{Eu}$ & $700-800$ & $\mathrm{O}_{3}$ \\
\hline
\end{tabular}


Table III. X-ray crystallographic data for $R E \mathrm{NiO}_{3}\left(R E=\mathrm{La}, \mathrm{Pr}\right.$, and Nd) films. The $a_{\mathrm{f}}$ and $c_{\mathrm{f}}$ were determined from positions of the (110) and (002) reflections in the $\omega-2 \theta$ scans, respectively. The $\Delta a_{\mathrm{f}}$ is calculated from the full width $(\Delta 2 \theta)$ at the half maximum of the (110) reflection. The $\Delta \omega$ and $\Delta \varphi$ denote the full width at the half maximum for the $\omega$ scans and $\varphi$ scans, respectively.

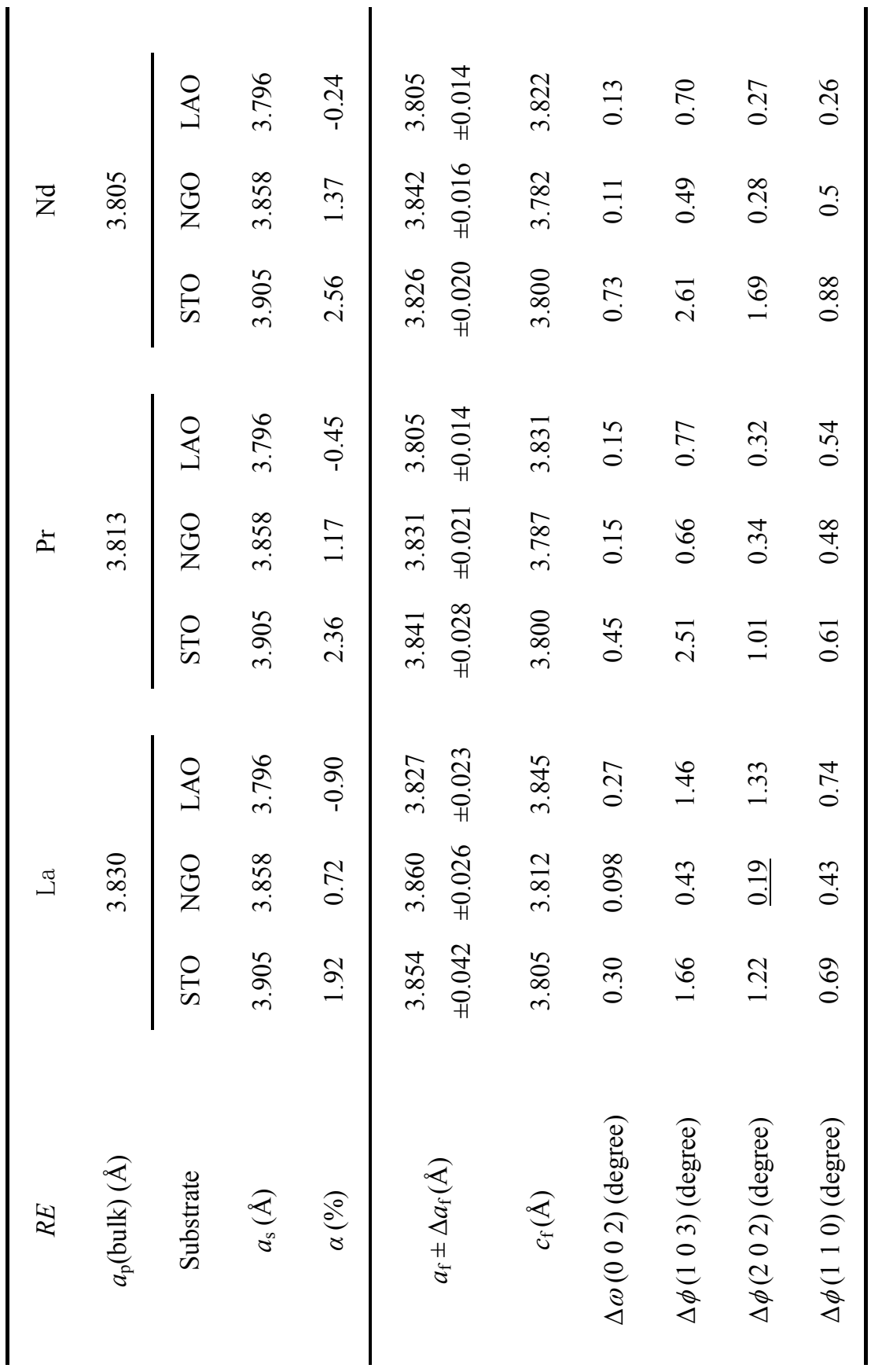


Table IV. The properties of $\mathrm{LaNiO}_{3}, \mathrm{PrNiO}_{3}$, and $\mathrm{NdNiO}_{3}$ films prepared by MOD (present work) and by other thin-film techniques. For reference, the data of bulk samples are also included.

\begin{tabular}{|c|c|c|c|c|c|}
\hline $\begin{array}{c}\text { Synthesis } \\
\text { method }\end{array}$ & Ref. & Material (thickness) & $\begin{array}{l}\rho(300 \mathrm{~K}) \\
(\mu \Omega \mathrm{cm})\end{array}$ & $R R R$ & $\begin{array}{l}T_{\mathrm{MI}} \\
(\mathrm{K}) \\
\end{array}$ \\
\hline MBE & 21 & $\mathrm{LaNiO}_{3} / \mathrm{STO}$ & 300 & 6 & - \\
\hline PLD & 22 & $\mathrm{LaNiO}_{3} / \mathrm{STO}$ & 500 & 2.4 & - \\
\hline Sputtering & 23 & $\mathrm{LaNiO}_{3} / \mathrm{LAO}$ & 130 & 8 & - \\
\hline MOD & this work & $\mathrm{LaNiO}_{3} / \mathrm{NGO}$ & 100 & 18 & - \\
\hline MOD & this work & $\mathrm{LaNiO}_{3} / \mathrm{LAO}$ & 130 & 16 & - \\
\hline MOD & this work & $\mathrm{LaNiO}_{3} / \mathrm{STO}$ & 140 & 10 & - \\
\hline bulk (cold press) & 15 & $\mathrm{LaNiO}_{3}$ & 100 & 15 & - \\
\hline $\mathrm{MBE}$ & 24 & $\mathrm{PrNiO}_{3}(120 \AA) / \mathrm{LAO}$ & 250 & 3.6 & 0 \\
\hline PLD & 11 & $\mathrm{PrNiO}_{3}(750 \AA) / \mathrm{LAO}$ & 74 & 2 & $0 *$ \\
\hline MOCVD & 25 & $\mathrm{PrNiO}_{3}(1000 \AA) / \mathrm{LAO}$ & 700 & - & 80 \\
\hline MOD & this work & $\mathrm{PrNiO}_{3} / \mathrm{NGO}$ & 440 & - & 125 \\
\hline MOD & this work & $\mathrm{PrNiO}_{3} / \mathrm{LAO}$ & 320 & 15 & 0 \\
\hline bulk & 8 & $\mathrm{PrNiO}_{3}$ & 500 & - & 130 \\
\hline PLD & 13 & $\mathrm{NdNiO}_{3} / \mathrm{NGO}$ & $(\sim 6 \Omega)^{* * *}$ & - & 170 \\
\hline PLD & 13 & $\mathrm{NdNiO}_{3} / \mathrm{LAO}$ & $(\sim 10 \Omega)^{* * *}$ & - & 120 \\
\hline PLD & 12 & $\mathrm{NdNiO}_{3}(200 \AA) / \mathrm{NGO}$ & 200 & - & 240 \\
\hline PLD & 26 & $\mathrm{NdNiO}_{3}(500 \AA) / \mathrm{NGO}$ & $(400)^{* *}$ & - & 160 \\
\hline Sputtering & 27 & $\mathrm{NdNiO}_{3}(120 \AA) / \mathrm{NGO}$ & 300 & - & 150 \\
\hline Sputtering & 27 & $\mathrm{NdNiO}_{3}(120 \AA) / \mathrm{LAO}$ & 300 & - & 80 \\
\hline MOD & this work & $\mathrm{NdNiO}_{3} / \mathrm{NGO}$ & 550 & - & 210 \\
\hline MOD & this work & $\mathrm{NdNiO}_{3} / \mathrm{LAO}$ & 400 & - & 140 \\
\hline bulk(cold press) & 15 & $\mathrm{NdNiO}_{3}$ & 300 & - & 200 \\
\hline bulk & 8 & $\mathrm{NdNiO}_{3}$ & 1100 & - & 200 \\
\hline
\end{tabular}

* Resistivity measurements were carried out in the limited temperature range $25-300 \mathrm{~K}$.

** Resistivity value at $200 \mathrm{~K}$.

*** No resistivity but resistance is presented in this article. 
Figure caption

Figure 1. Structural and transport data for $R E \mathrm{NiO}_{3}$ films prepared under various growth parameters: (a) variation of the (002) peak intensity and (b) $\rho(295 \mathrm{~K})$ as a function of firing temperature, $T_{\mathrm{f}}$. The films were prepared on NGO at various $T_{\mathrm{f}}=650-900^{\circ} \mathrm{C}$ under either $\mathrm{O}_{2}$ or $\mathrm{O}_{3}$ flow.

Figure 2. Temperature dependence of resistivity for $\mathrm{PrNiO}_{3}$ films on $\mathrm{NGO}$ fired at various temperature $T_{\mathrm{f}}=650-850^{\circ} \mathrm{C}$ in an $\mathrm{O}_{3}$ flow.

Figure 3. (a) XRD patterns and (b) $\rho-T$ curves of the $R E \mathrm{NiO}_{3}$ films $(R E=\mathrm{La}, \mathrm{Pr}, \mathrm{Nd}, \mathrm{Sm}$, and $\mathrm{Eu}$ ) on NGO obtained after optimizing synthesis conditions. In the XRD patterns, all peaks can be indexed for the $(00 l)$ reflections of the perovskite structure.

Figure 4. $c$-axis length $\left(c_{\mathrm{f}}\right)$ of $R E \mathrm{NiO}_{3}$ films $(R E=\mathrm{La}, \mathrm{Pr}, \mathrm{Nd}, \mathrm{Sm}$, and $\mathrm{Eu})$ grown on $\mathrm{NGO}$, LAO, and STO plotted against the ionic size $r_{\mathrm{i}}^{\mathrm{XII}}\left(R E^{3+}\right)$ of $R E$. The open circles represent the bulk $a_{\mathrm{p}}$ (bulk) of $R E \mathrm{NiO}_{3}$.

Figure 5. $\phi$ scan of the (103) reflection of the $\mathrm{PrNiO}_{3}$ film on NGO: (a) overall view and (b) enlarged view for a single peak.

Figure 6. $\omega-2 \theta$ scans of the (110) reflection for the $\mathrm{PrNiO}_{3}$ films on (a) $\mathrm{NGO}$, (b) LAO, and (c) STO, respectively. The substrate peak can be seen together with the film peak, but its intensity depends on the alignment. The substrate peak is large on NGO whereas it is only discernible on LAO. The dotted line in (a) is a guide to the eye. The arrows indicate the position of the (110) reflection expected for a bulk specimen. 
Figure 7. Hysteresis of the $\rho-T$ curve for the $\mathrm{NdNiO}_{3}$ film on $\mathrm{NGO}$ measured on cooling (open circles) and heating (solid circles). The transition hysteresis, $\Delta T_{\mathrm{MI}}$, on cooling and heating is $\leq 10 \mathrm{~K}$, indicating that our $\mathrm{NdNiO}_{3}$ films may be orthorhombic.

Figure 8. Comparison of $\rho-T$ curves of the $R E \mathrm{NiO}_{3}$ films $(R E=\mathrm{La}, \mathrm{Pr}, \mathrm{Nd}$, and $\mathrm{Sm})$ on LAO, NGO, and STO. 
Fig. 1 Ikeda et al
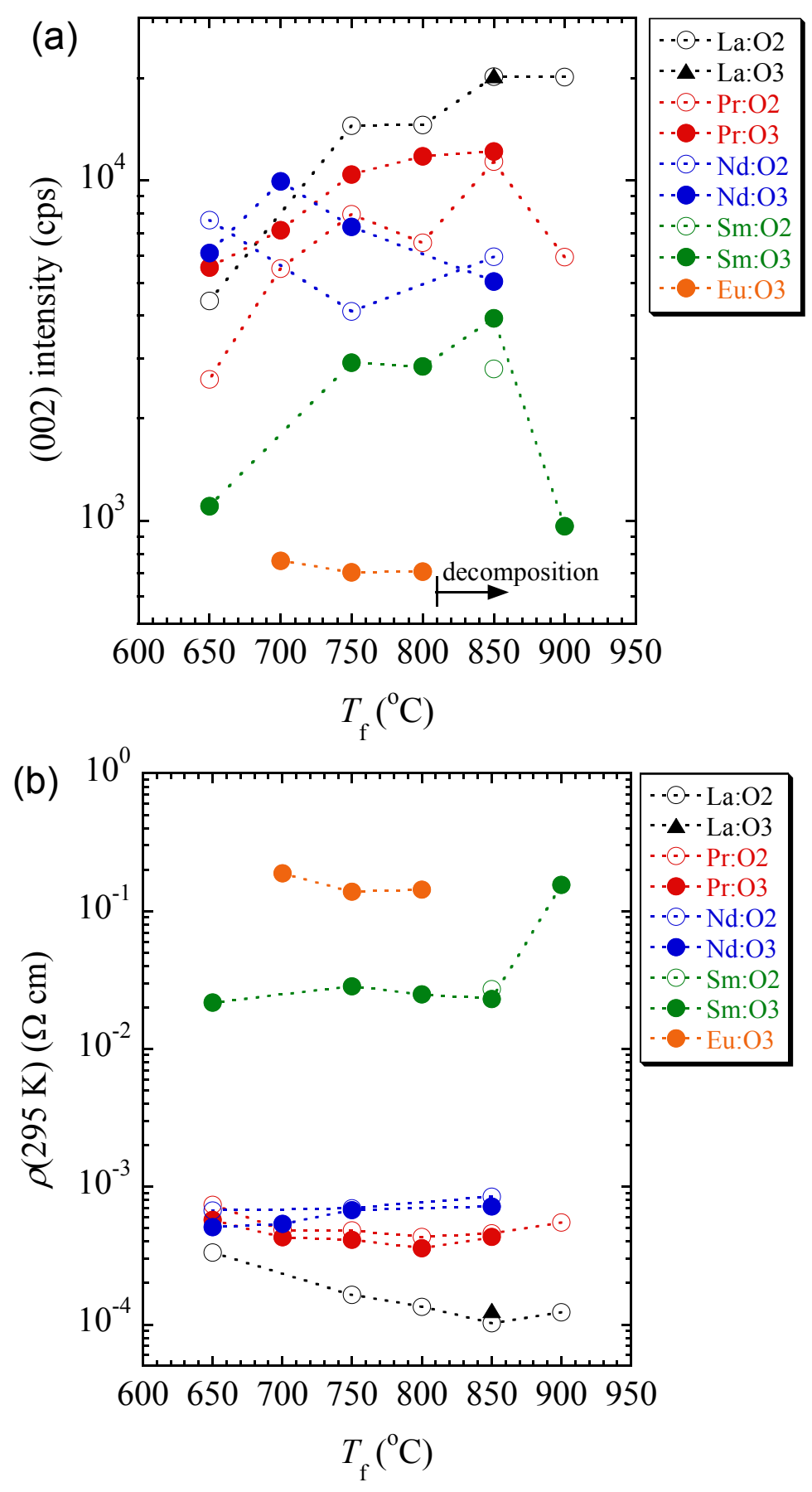
Fig. 2 Ikeda et al

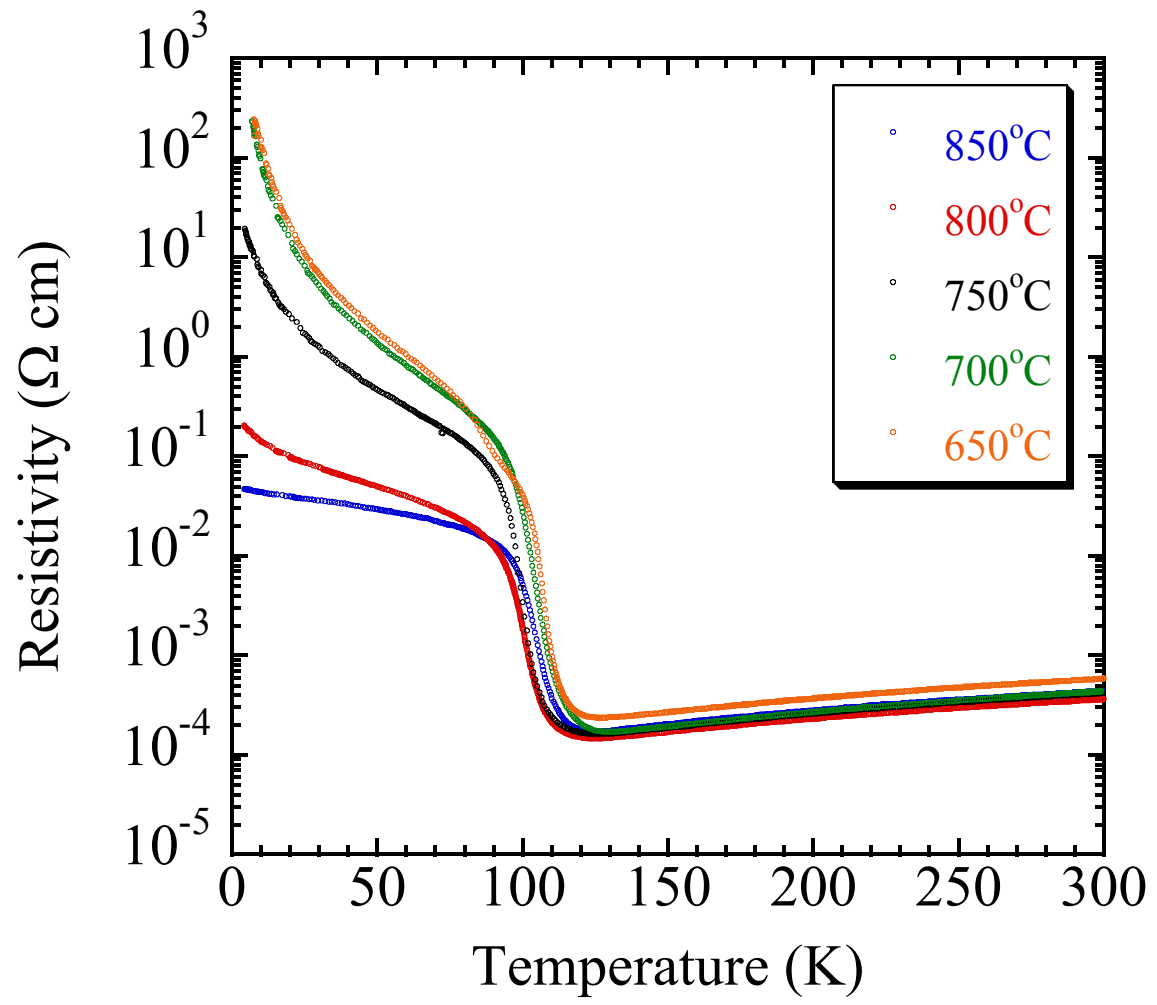


Fig. 3 Ikeda et al
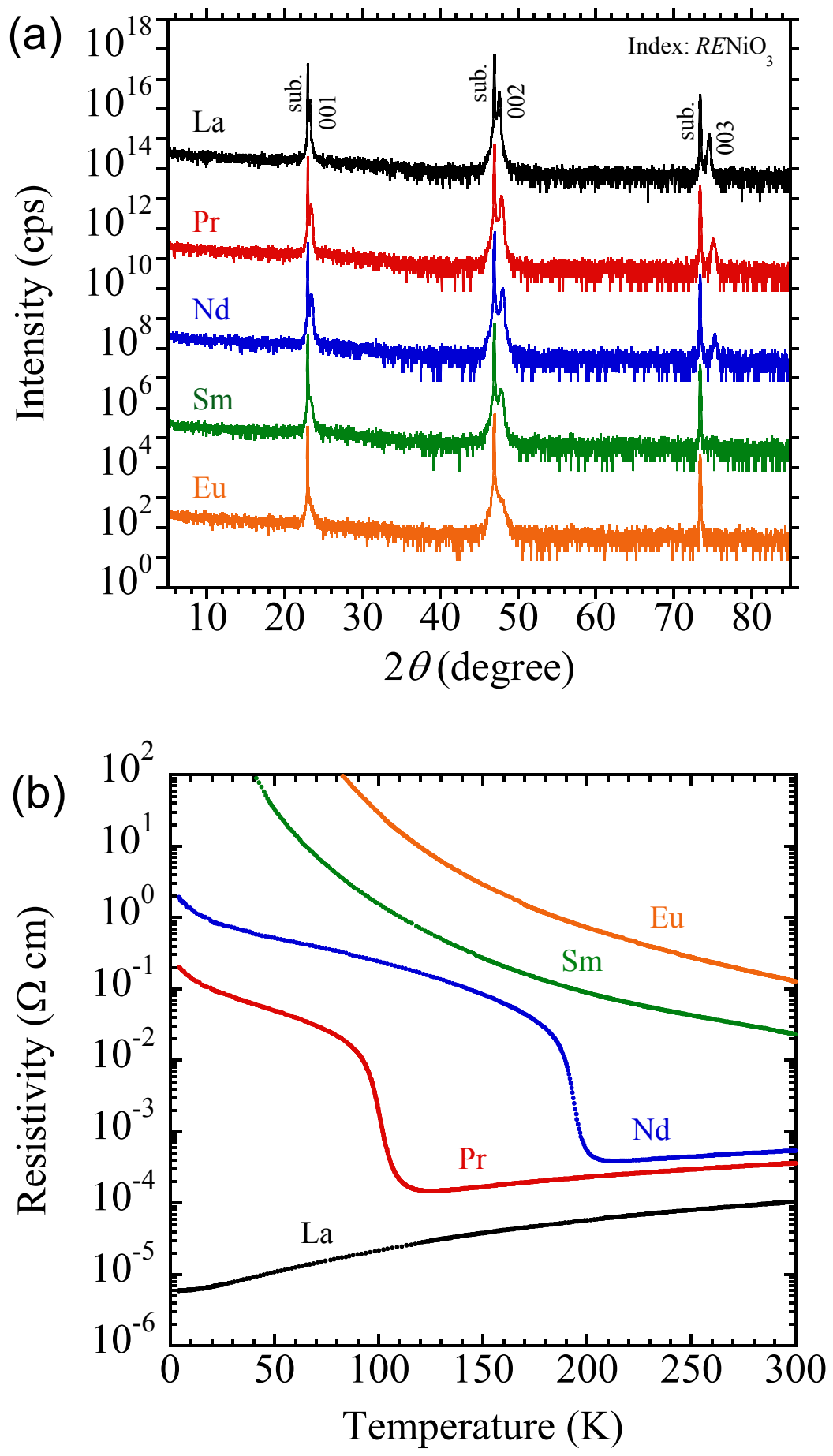
Fig. 4 Ikeda et al

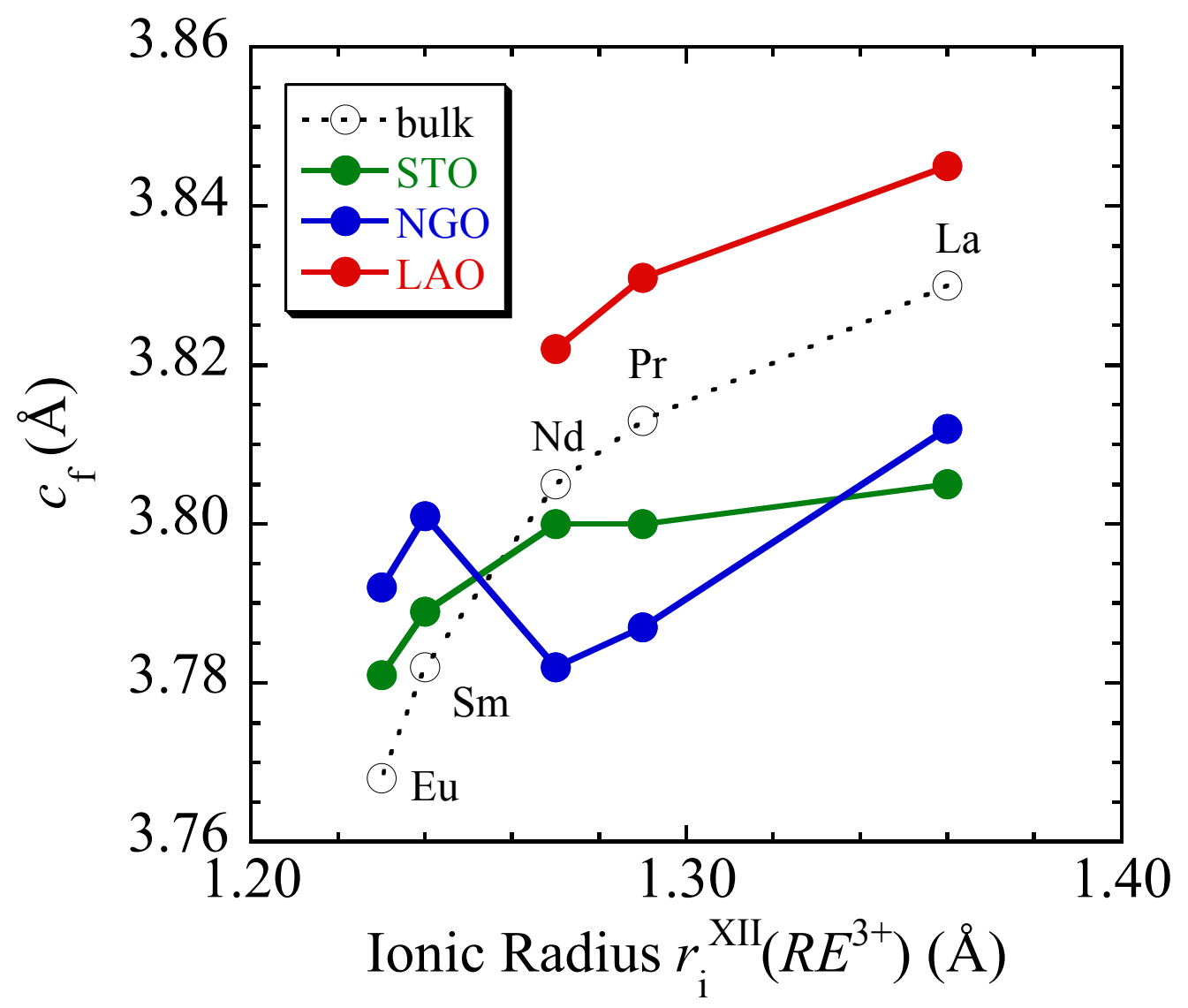


Fig. 5 Ikeda et al
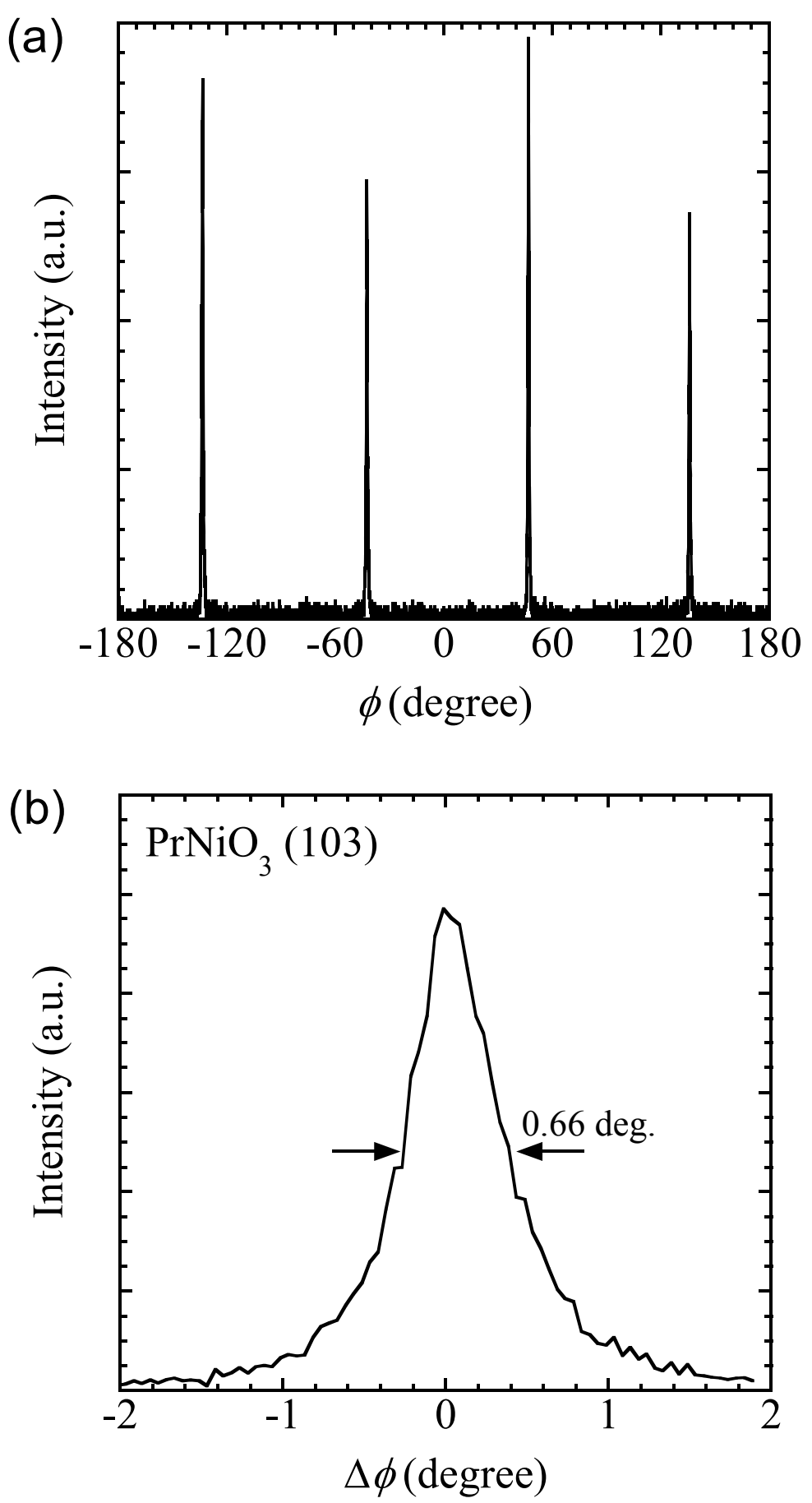
Fig. 6 Ikeda et al
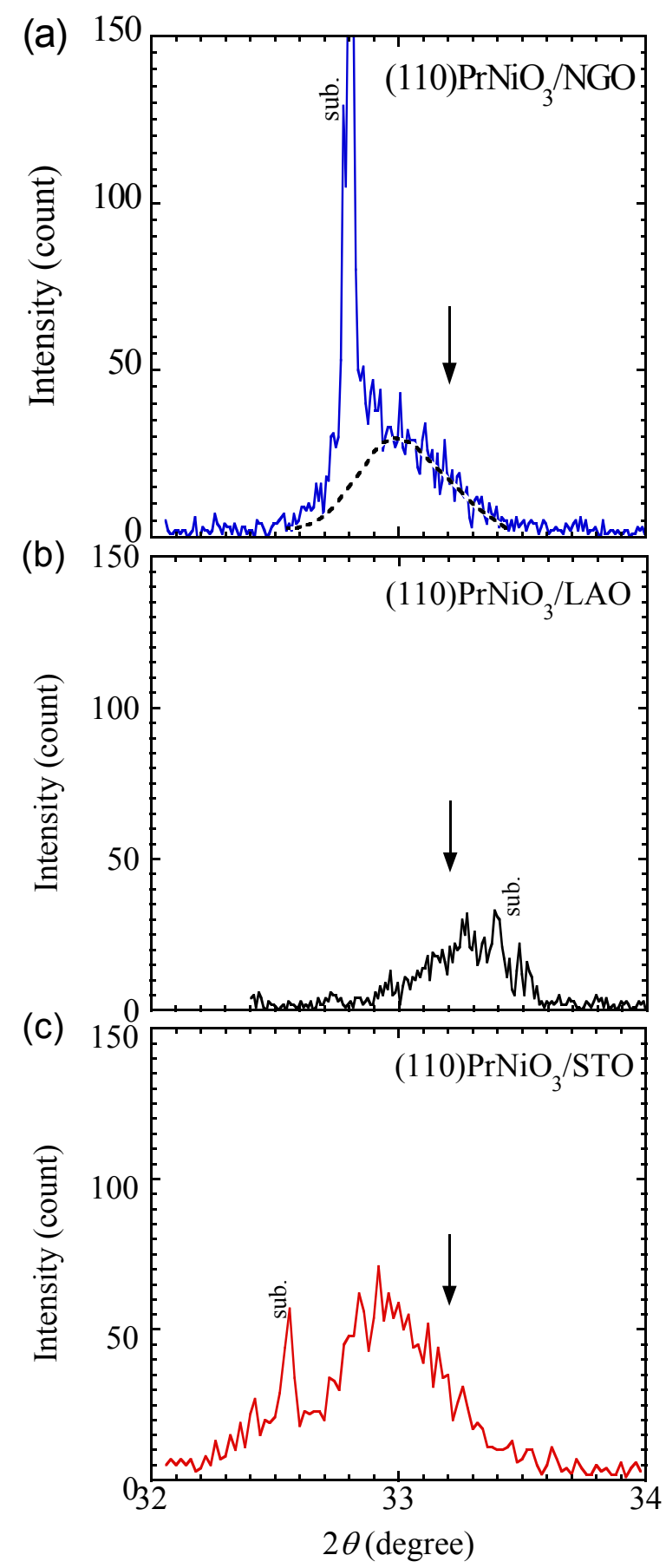
Fig. 7 Ikeda et al

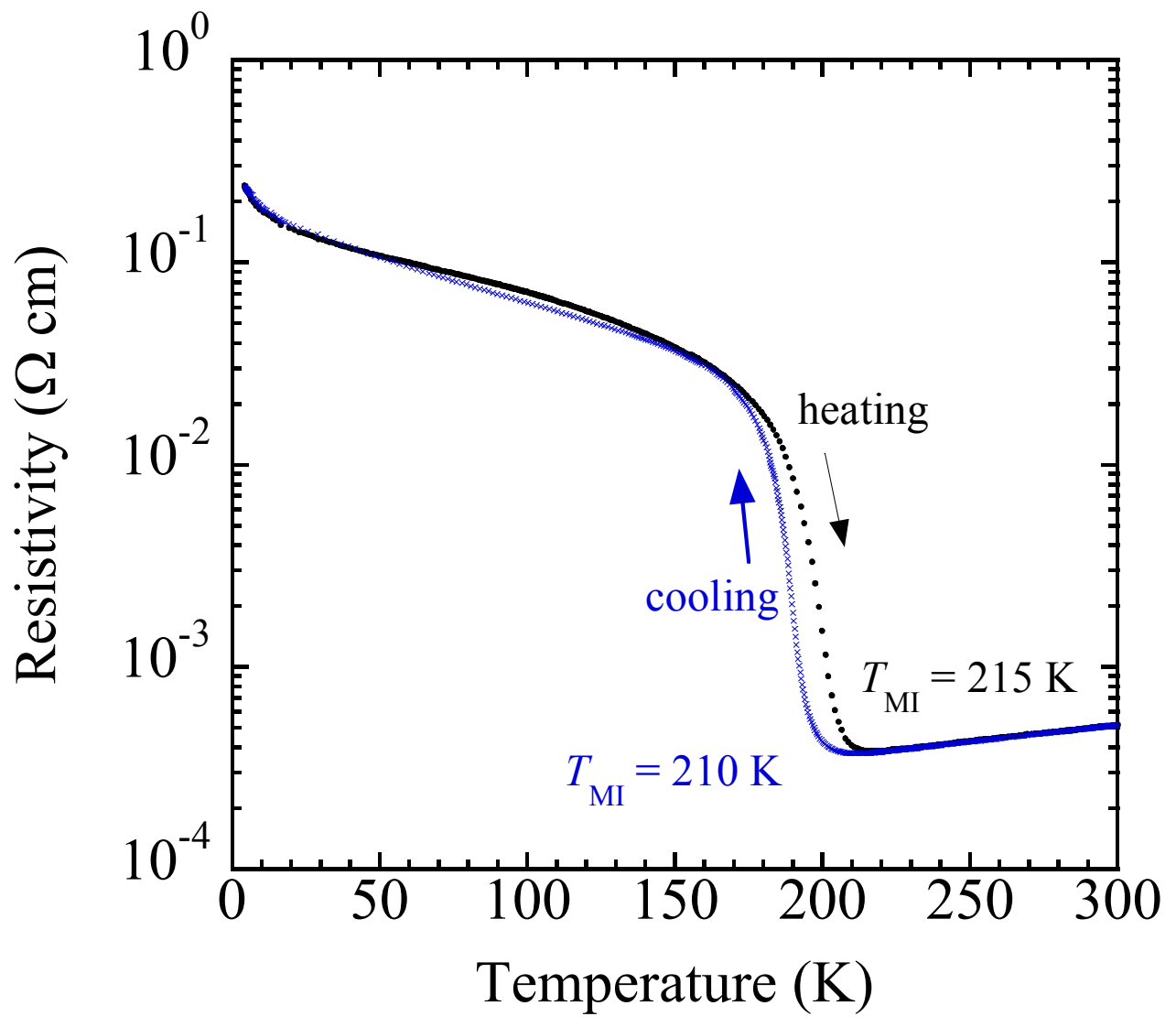


Fig. 8 Ikeda et al
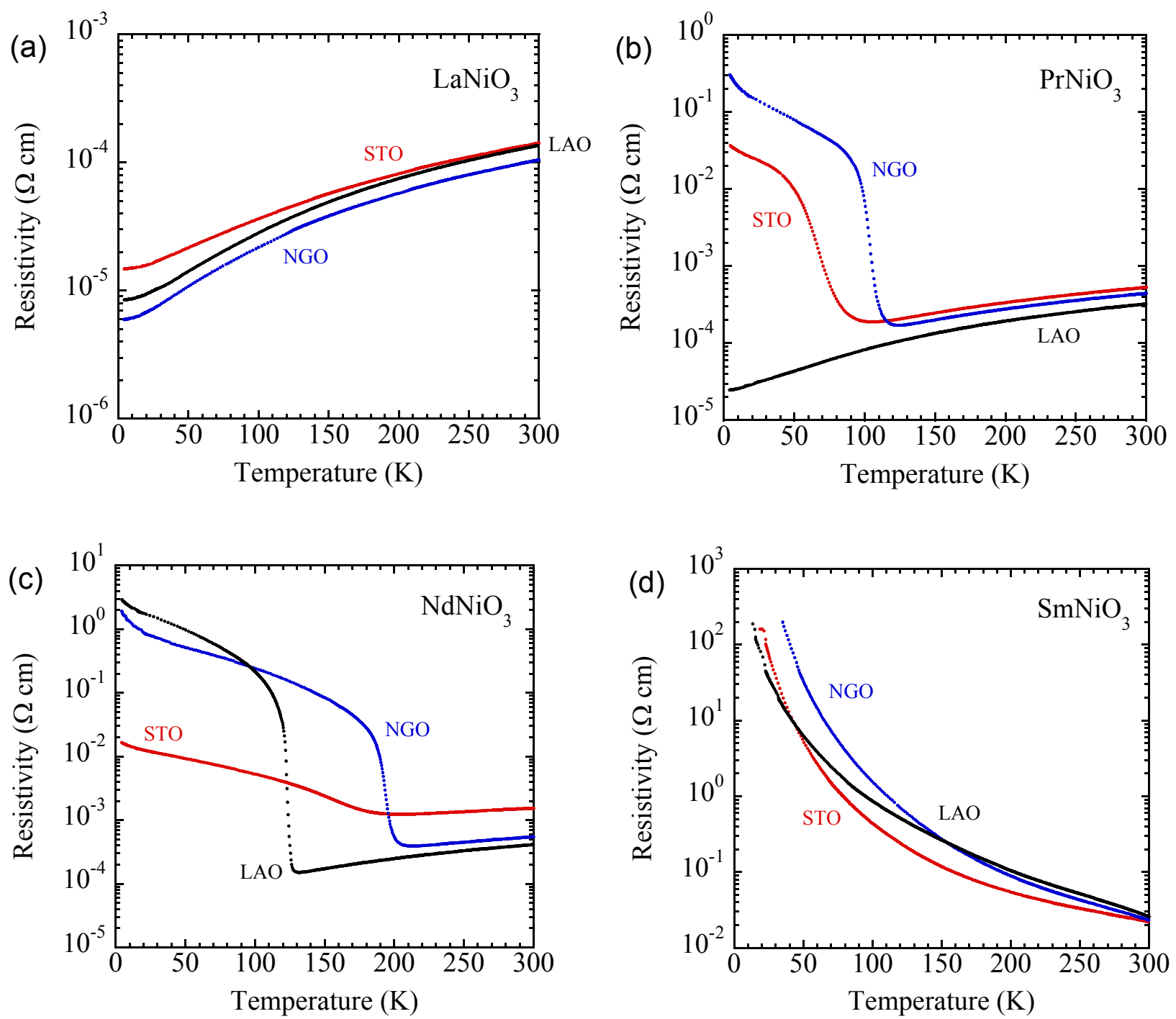